Internat. J. Math. \& Math. Sci.

Vol. 24, No. 6 (2000) 371-377

S0161171200004336

(C) Hindawi Publishing Corp.

\title{
EXISTENCE OF PERIODIC TRAVELING WAVE SOLUTION TO THE FORCED GENERALIZED NEARLY CONCENTRIC KORTEWEG-DE VRIES EQUATION
}

\author{
KENNETH L. JONES, XIAOGUI HE, and YUNKAI CHEN
}

(Received 15 October 1999)

\begin{abstract}
This paper is concerned with periodic traveling wave solutions of the forced generalized nearly concentric Korteweg-de Vries equation in the form of $\left(u_{\eta}+u /(2 \eta)+\right.$ $\left.[f(u)]_{\xi}+u_{\xi \xi \xi}\right)_{\xi}+u_{\theta \theta} / \eta^{2}=h_{0}$. The authors first convert this equation into a forced generalized Kadomtsev-Petviashvili equation, $\left(u_{t}+[f(u)]_{x}+u_{x x x}\right)_{x}+u_{y y}=h_{0}$, and then to a nonlinear ordinary differential equation with periodic boundary conditions. An equivalent relationship between the ordinary differential equation and nonlinear integral equations with symmetric kernels is established by using the Green's function method. The integral representations generate compact operators in a Banach space of real-valued continuous functions. The Schauder's fixed point theorem is then used to prove the existence of nonconstant solutions to the integral equations. Therefore, the existence of periodic traveling wave solutions to the forced generalized KP equation, and hence the nearly concentric KdV equation, is proved.
\end{abstract}

Keywords and phrases. Existence theorem, traveling wave solution, forced generalized nearly concentric Korteweg-de Vries equation.

2000 Mathematics Subject Classification. Primary 35B10, 35B20, 76B15.

1. Introduction. The purely concentric or cylindrical Korteweg-de Vries equation (KdV equation for short),

$$
u_{\eta}+\frac{u}{2 \eta}+u u_{\xi}+u_{\xi \xi \xi}=0
$$

was first derived by Maxon and Viecelli in 1974 from the study of propagation of radically ingoing acoustic waves in cylindrical geometry [9]. In this equation, $u=$ $u(\eta, \xi), \eta=\varepsilon^{3 / 2} \omega_{i} t$, and $\xi=-\varepsilon^{1 / 2}\left(r / \lambda_{D}+\omega_{i} t\right)$, where $\varepsilon$ is the expansion parameter, $\lambda_{D}$ the Debye length, $\omega_{i}$ the ion plasma frequency, $r$ the radial distance, and $t$ the time. In [6], Johnson generalized the purely concentric KdV equation to the following nearly concentric KdV equation by considering the nearly straight wave propagation which varies in a very small angular region

$$
\left(u_{\eta}+\frac{u}{2 \eta}+u u_{\xi}+u_{\xi \xi \xi}\right)_{\xi}+\frac{u_{\theta \theta}}{\eta^{2}}=0
$$

where $u=u(\eta, \xi, \theta)$ and $\theta$ is the angular variable which varies in a small region [6, 7]. For the general review of this equation and some subsequent developments, the authors cite the book by Infeld and Rowland [5]. 
In this paper, the authors consider the KdV equation of the form

$$
\left(u_{\eta}+\frac{u}{2 \eta}+[f(u)]_{\xi}+u_{\xi \xi \xi}\right)_{\xi}+\frac{u_{\theta \theta}}{\eta^{2}}=h_{0},
$$

where $f$ is a $C^{2}$ function of its argument and $h_{0}$ is a nonconstant function of $\eta$, $\xi$, and $\theta$. This equation is a generalization of Johnson's equation and also a nearly concentric version of the forced KdV equation obtained by Akylas [1], Wu [14], and Shen [10, 11]. We call this equation forced generalized nearly concentric KdV equation.

The authors convert the equation into the forced generalized Kadomtsev-Petviashvili equation (referred to as KP equation henceforth) in the form

$$
\left(u_{t}+[f(u)]_{x}+u_{x x x}\right)_{x}+u_{y y}=h_{0} .
$$

Following the idea of Liu and Pao [8], Soewono [12], and Chen and He [4], the authors will use the Green's function method to derive nonlinear integral equations from (1.4), which are equivalent to the generalized forced KP equation with periodic boundary conditions. Imposing suitable conditions, the authors shall establish the existence of nonconstant solutions to the integral equations. And hence, prove the existence of periodic traveling wave solutions to (1.3) and (1.4). Furthermore, we note that the nonconstant periodic traveling wave solutions are infinitely differentiable.

The content of the paper is arranged as follows. In Section 2, the author convert the forced generalized nearly concentric KdV equation to a forced generalized KP equation and then to nonlinear integral equations using the Green's function method. Section 3 contains the proof of the existence of nonconstant solutions to these integral equations. Therefore, the existence of periodic traveling wave solutions to the forced generalized KP equation, and hence the forced generalized nearly concentric KdV equation, is established.

2. Formulation of the problem. We start from the forced generalized nearly concentric KdV equation

$$
\left(u_{\eta}+\frac{u}{2 \eta}+[f(u)]_{\xi}+u \xi \xi \xi\right)_{\xi}+\frac{u_{\theta \theta}}{\eta^{2}}=h_{0}
$$

where $f$ is a $C^{2}$ function of its argument and $h_{0}$ is a nonconstant function of $\eta, \xi$, and $\theta$. Considering that (2.1) is more analogous to the two-dimensional case and motivated by the results obtained by Chen [2, 3], the authors introduce the transformations $\eta=t$, $\xi=x+y / 4 t$, and $\theta=y / x$. One can argue that since $\tan \theta=y / x$ and $\theta$ is the variable in a very small angular sector, $\theta$ can be used to approximate $y / x$. Thus when $x$ and $t$ are large and of the same order, it seems to be reasonable to assume $\theta=y / x$. Use $u(x, y, t)$ to replace $u(\eta, \xi, \theta)$, then we shall have

$$
\begin{gathered}
u_{t x}=u_{\eta \xi}-\frac{y^{2}}{4 \eta^{2}} u_{\xi \xi}, \quad f(u)_{x}=f(u)_{\xi} \\
u_{x x x x}=u_{\xi \xi \xi \xi}, \quad u_{y y}=\frac{y^{2}}{4 \eta^{2}} u_{\xi \xi}+\frac{1}{2 \eta} u_{\xi}+\frac{1}{\eta^{2}} u_{\theta \theta} .
\end{gathered}
$$


Therefore, equation (2.1) can be converted to the forced generalized KP equation of the form

$$
\left(u_{t}+[f(u)]_{x}+u_{x x x}\right)_{x}+u_{y y}=h_{0}
$$

where $f$ is a $C^{2}$ function of its argument and $h_{0}$ is a nonconstant function of $x, y$, and $t$. We are interested in the periodic traveling wave solutions of the form $U(z)=$ $u(x, y, t)$, where $z=a x+b y-\omega t$ with $a, b$, and $\omega$ being real constants. Without loss of generality, we assume $a>0$. In this paper, we only consider the case that $h_{0}(x, y, t)=a^{2} h(z)$ is a $2 T$-periodic continuous function of $z$ with a preassigned positive number $T$. Substitution of the $U(z)$ and $a^{2} h(z)$ into (2.3) leads to the fourth order nonlinear ordinary differential equation

$$
U^{(4)}(z)=\frac{C}{a^{2}} U^{\prime \prime}(z)-\frac{1}{a^{2}}\left[f(U(z))\left(U^{\prime}(z)\right)^{2}+f^{\prime}(U(z)) U^{\prime \prime}(z)\right]+\frac{1}{a^{2}} h(z),
$$

where $C=\left(\omega a-b^{2}\right) / a^{2}$. We now impose the following periodic boundary conditions

$$
U^{(n)}(0)=U^{(n)}(2 T), \quad n=0,1,2,3 .
$$

In addition, to rule out nonzero constant solutions, another condition is introduced,

$$
\int_{0}^{2 T} U(z) d z=0
$$

It can be seen that any solution of the boundary value problem consisting of (2.4), (2.5), and (2.6) can be extended to a $2 T$-periodic traveling wave solution to (2.3).

Integrating both sides of (2.4) with respect to $z$ twice and using (2.5) and (2.6) yields

$$
\begin{gathered}
U^{\prime \prime}(z)-\frac{C}{a^{2}} U(z)=E-\frac{1}{a^{2}}[f(U(z))-H(z)], \\
U^{(n)}(0)=U^{(n)}(2 T), \quad n=0,1,
\end{gathered}
$$

where

$$
E=\left(\frac{1}{\left(2 T a^{2}\right)}\right) \int_{0}^{2 T}[f(U(z))-H(z)] d z,
$$

and $H(z)$ is a $2 T$-periodic function of $z$ such that $H^{\prime \prime}(z)=h(z)$. Conversely, integrating both sides of (2.7) from zero to $2 T$ and using (2.8) will lead to (2.6), and direct differentiations of (2.7) will give us (2.4) and (2.5). Therefore, we have proved the following theorem by noting from (2.7) that $U \in C^{2}[0,2 T]$ implies $U \in C^{4}[0,2 T]$ since $f$ is a $C^{2}$ function of its argument.

THEOREM 2.1. Suppose $C \neq 0$, a function $U(z)$ is a solution of the boundary value problem (2.4), (2.5), and (2.6) if and only if it is a solution of the boundary value problem (2.7) and (2.8).

From now on we only consider the two cases: (1) $C>0$ and (2) $C<0$ but $-C / a^{2} \neq$ $(k \pi / T)^{2}$ with $k$ being any integer. Denote the function $f(U(z))-H(z)$ on the righthand side of (2.7) by $F(U(z))$. Treating the right-hand side of (2.7) as a forcing term 
and using the Green's function method [13], the boundary value problem (2.7) and (2.8) can be converted to two integral equations

$$
U(z)=\frac{1}{a^{2}} \int_{0}^{2 T} K_{i}(z, s) F(U(s)) d s,
$$

where the kernels $K_{i}, i=1,2$, are defined as follows:

(1) When $C>0$, let $\lambda_{1}=\sqrt{C / a^{2}}$, then

$$
K_{1}(z, s)=\frac{\cosh \lambda_{1}(T-|z-s|)}{2 \lambda_{1} \sinh \lambda_{1} T}-\frac{1}{2 \lambda_{1}^{2} T}, \quad \forall z, s \in[0,2 T] .
$$

(2) When $C<0$ but $-C / a^{2} \neq(k \pi / T)^{2}$ with $k$ being any integer, let $\lambda_{2}=\sqrt{-C / a^{2}}$, then

$$
K_{2}(z, s)=\frac{\cos \lambda_{2}(T-|z-s|)}{2 \lambda_{2} \sin \lambda_{2} T}-\frac{1}{2 \lambda_{2}^{2} T}, \quad \forall z, s \in[0,2 T] .
$$

LEMMA 2.2. The kernels $K_{1}$ and $K_{2}$ have the following properties:

$$
\begin{aligned}
K_{i}(0, s) & =K_{i}(2 T, s), \quad \forall s \in[0,2 T], i=1,2, \\
K_{i}(z, 2 T-s) & =K_{i}(2 T-z, s), \quad \forall z, s \in[0,2 T], i=1,2 .
\end{aligned}
$$

PROoF. Straightforward computations from the definitions of the kernels $K_{i}, i=$ 1,2 , given in (2.11) and (2.12).

THEOREM 2.3. A function $U(z)$ is a solution of the boundary value problem (2.7) and (2.8) if and only if it is a solution of the integral equation (2.10).

Proof. The if part can be proved by direct differentiations of (2.10) and the only if part is based on the Green's function method by treating the right-hand side of (2.7) as a nonhomogeneous term.

3. Existence theorem. To show the existence of $2 T$-periodic traveling wave solutions to (2.1) it is sufficient to show that solutions to (2.10) exist.

To this end we define $C_{2 T}$ as a collection of real-valued continuous functions, $v(z)$, on $[0,2 T]$ such that $v(0)=v(2 T)$. Equip $C_{2 T}$ with the sup norm $\|\cdot\|$ as $\|v\|=$ $\sup _{0 \leq z \leq 2 T}|v(z)|$, for each $v \in C_{2 T}$. Then $\left(C_{2 T},\|\cdot\|\right)$ is a Banach space.

We define operators $\mathscr{A}_{i}, i=1,2$, on $C_{2 T}$ as

$$
\mathscr{A}_{i} v(z)=\frac{1}{a^{2}} \int_{0}^{2 T} K_{i}(z, s) F(v(s)) d s, \quad \forall v \in C_{2 T},
$$

where the kernels $K_{i}, i=1,2$, are given in (2.11) and (2.12). We shall demonstrate that there exist functions $v$ in $C_{2 T}$ such that $v=\mathscr{A}_{i} v, i=1$, 2, and hence, prove that there exist solutions to (2.10).

Let

$$
\begin{gathered}
Q_{i} \geq \max _{0 \leq z \leq 2 T} \int_{0}^{2 T}\left|K_{i}(z, s)\right| d s, \quad i=1,2, \\
\tau_{1}=1, \quad \tau_{2}=\left|\sin \lambda_{2} T\right| .
\end{gathered}
$$

A consequence of Lemma 2.2 can now be stated. 
LEMMA 3.1. Let $v$ be an element of $C_{2 T}$. If $v(z)=v(2 T-z)$ for $z \in[0,2 T]$, then $\mathscr{A}_{i} v(z)=\mathscr{A}_{i} v(2 T-z), i=1,2$.

We now define $B(0, r)$ to be a closed ball in $C_{2 T}$ and let $M=\sup [\|F(v)\|: v \in B(0, r)]$. We have the following existence theorem.

THEOREM 3.2. Let $\mathscr{A}_{i}, i=1,2$, be a compact operator from $C_{2 T}$ to $C_{2 T}$. In particular, if $Q_{i} M / a^{2} \leq r, i=1,2$, then $\mathscr{A}_{i}$ maps $B(0, r)$ into itself. Hence, the integral equation (2.10) has at least one solution in $B(0, r)$.

Proof. First we show $\mathscr{A}_{i}: C_{2 T} \rightarrow C_{2 T}, i=1,2$. Since it is obvious from Lemma 2.2 that $\mathscr{A}_{i} v(0)=\mathscr{A}_{i} v(2 T)$ for each $v \in C_{2 T}, i=1,2$, it suffices to show that $\mathscr{A}_{i} v, i=1,2$, is continuous on $[0,2 T]$.

Let $v$ be an arbitrary function in $C_{2 T}$, we have

$$
\begin{aligned}
\frac{d \mathscr{A}_{1} v(z)}{d z}= & \frac{-1}{2 a^{2} \sinh \lambda_{1} T} \int_{0}^{z} \sinh \lambda_{1}(T-z+s) F(v(s)) d s \\
& +\frac{1}{2 a^{2} \sinh \lambda_{1} T} \int_{z}^{2 T} \sinh \lambda_{1}(T+z-s) F(v(s)) d s, \\
\frac{d A_{2} v(z)}{d z}= & \frac{1}{2 a^{2} \sin \lambda_{2} T} \int_{0}^{z} \sin \lambda_{2}(T-z+s) F(v(s)) d s \\
& +\frac{-1}{2 a^{2} \sin \lambda_{2} T} \int_{z}^{2 T} \sin \lambda_{2}(T+z-s) F(v(s)) d s .
\end{aligned}
$$

The existence of $d \mathscr{A}_{1} v / d z$ and $d \mathscr{A}_{2} v / d z$ implies that both $\mathscr{A}_{1} v$ and $\mathscr{A}_{2} v$ are continuous on $[0,2 T]$, and hence, $\mathscr{A}_{i}: C_{2 T} \rightarrow C_{2 T}, i=1,2$.

Let $\mathbf{S}$ be a bounded subset of $C_{2 T}$, i.e., there exists an $L_{0}>0$ such that $\|v\|<L_{0}$ for all $v \in \mathbf{S}$. Then there must be an $M_{0}>0$ such that

$$
\|F(v)\|=\sup _{0 \leq z \leq 2 T}|F(v(z))| \leq \sup _{-L_{0} \leq w \leq L_{0}}|F(w)| \leq M_{0}, \quad \forall v \in \mathbf{S} .
$$

Thus from (3.1), (3.3), and (3.4) we have

$$
\begin{aligned}
\left\|\mathscr{A}_{i} v\right\| & \leq \frac{1}{a^{2}} Q_{i} M_{0}, \quad \forall v \in \mathbf{S}, i=1,2, \\
\left\|\frac{d \mathscr{A}_{i} v}{d z}\right\| & \leq \frac{T}{a^{2} T_{i}} M_{0}, \quad \forall v \in \mathbf{S}, i=1,2 .
\end{aligned}
$$

Therefore, $\mathscr{A}_{i} \mathbf{S}, i=1,2$, is uniformly bounded and equi-continuous, and by the Ascoli-Arzela theorem both $\mathscr{A}_{1}$ and $\mathscr{A}_{2}$ are compact operators from $C_{2 T}$ to $C_{2 T}$.

To show that $\mathscr{A}_{i}, i=1,2$, has a fixed point in $B(0, r)$ when $Q_{i} M / a^{2} \leq r, i=1,2$, we write

$$
\begin{aligned}
\left|\mathscr{A}_{i} v(z)\right| & =\frac{1}{a^{2}}\left|\int_{0}^{2 T} K_{i}(z, s) F(v(s)) d s\right| \\
& \leq \frac{1}{a^{2}} \int_{0}^{2 T}\left|K_{i}(z, s)\right||F(v(s))| d s \\
& \leq \frac{Q_{i} M}{a^{2}} \leq r, \quad \forall v \in B(0, r) .
\end{aligned}
$$


This implies that $\left\|\mathscr{A}_{i} v\right\| \leq r$ for all $v \in B(0, r), i=1,2$, and hence, $\mathscr{A}_{i}, i=1,2$, maps $B(0, r)$ into itself. Therefore, by the Schauder's fixed point theorem we proved that $\mathscr{A}_{i}$ has a fixed point in $B(0, r)$ for each $i=1,2$. And hence, equation (2.10) has a solution for each case of $C>0$ and $C<0$ with $-C /\left(\alpha a^{2}\right) \neq(k \pi / T)^{2}$.

It is worth noting that as long as $\int_{0}^{2 T} K_{i}(z, s) H(s) d s \neq 0, i=1,2$, by Theorem 3.2, there exists a nonconstant function $v(z)$ on $[0,2 T]$ such that $v=\mathscr{A}_{i} v, i=1,2$, which implies that $v(z)$ is infinitely differentiable on $[0,2 T]$ since $\mathscr{A}_{i} v$ is differentiable on $[0,2 T]$. The extension of the $v(z)$ to a $2 T$-periodic function $V(z)$ provides an infinitely differentiable $2 T$-periodic traveling wave solution to the forced generalized $\mathrm{KP}$ equation, and hence, a $2 T$-periodic traveling wave solution to the forced generalized nearly concentric KdV equation.

\section{REFERENCES}

[1] T. R. Akylas, On the excitation of long nonlinear water waves by a moving pressure distribution, J. Fluid Mech. 141 (1984), 455-466. MR 85c:76013. Zbl 551.76018.

[2] Y. Chen, Cylindrical Korteweg-de Vries equation and traveling wave solutions, J. Tech. Phys. 38 (1997), no. 1, 37-42. MR 98c:35138.

[3] _ Nearly concentric Korteweg-de Vries equation and periodic traveling wave solution, Internat. J. Math. Math. Sci. 21 (1998), no. 1, 183-188. MR 98i:35163. Zbl 895.35090.

[4] Y. Chen and X. He, Nonexistence of small periodic traveling wave solutions to the power Kadomtsev-Petviashvili equation, Differential Equations Dynam. Systems 5 (1997), no. 1, 43-50. MR 99h:35183. Zbl 891.34021.

[5] E. Infeld and G. Rowlands, Nonlinear Waves, Solitons and Chaos, Cambridge University Press, Cambridge, 1990. MR 92c:76001. Zbl 726.76018.

[6] R. S. Johnson, Water waves and Korteweg-de Vries equations, J. Fluid Mech. 97 (1980), no. 4, 701-719. MR 82d:76011. Zbl 441.76012.

[7] _ The Korteweg-de Vries equation and related problems in water wave theory, Nonlinear Waves (Greenville, N.C., 1982) (Cambridge), Cambridge Monographs Mech. Appl. Math., Cambridge Univ. Press, 1983, pp. 25-43. MR 85j:76008. Zbl 571.76011.

[8] B.-P. Liu and C. V. Pao, Green's function method for periodic traveling wave solutions of the KdV equation, Applicable Anal. 14 (1983), no. 4, 293-301. MR 85e:35014. Zbl 515.35077.

[9] S. Maxon and J. Viecelli, Cylindrical solitons, Physics of Fluids 39 (1974), 1614-1616.

[10] S. S. P. Shen, Disturbed critical surface waves in a channel of arbitrary cross section, Z. Angew. Math. Phys. 40 (1989), no. 2, 216-229. MR 90g:76032. Zbl 668.76018.

[11] On the accuracy of the stationary forced Korteweg-de Vries equation as a model equation for flows over a bump, Quart. Appl. Math. 53 (1995), no. 4, 701-719. MR 96i:76019. Zbl 859.76008.

[12] E. Soewono, A remark on a paper by Liu and Pao on the existence of periodic traveling wave solution to the KdV equation, Appl. Anal. 25 (1987), no. 4, 293-299. MR 89d:35171. Zbl 609.35079.

[13] I. Stakgold, Green's Functions and Boundary Value Problems, John Wiley \& Sons, New York, Chichester, Brisbane, 1979. MR 80k:35002. Zbl 421.34027.

[14] T. Y. Wu, Generation of upstream advancing solitons by moving disturbances, J. Fluid Mech. 184 (1987), 75-99. MR 89b:76008. Zbl 644.76017.

Kenneth L. Jones: Department of Mathematics and Computer SCience, FayetTeville StATE University, FAyeTteVIlle, North CAROLINA 28301-4298, USA

E-mail address: kjones@uncfsu.edu 


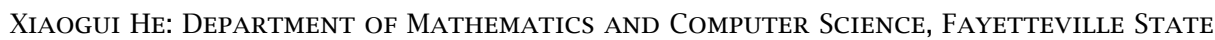
UNIVERSITY, FAYETTEVILLE, NORTH CAROLINA 28301-4298, USA

E-mail address: hchen@uncfsu.edu

Yunkai Chen: Department of MATHematics AND Computer SCienCe, FAYETTEVILle STATE UNIVERSITY, FAYETTEVILLE, NORTH CAROLINA 28301-4298, USA

E-mail address: ychen@uncfsu. edu 


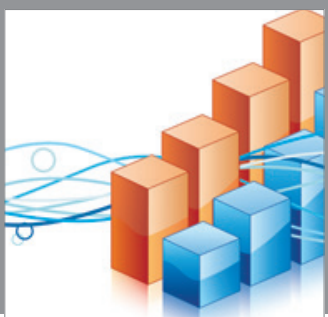

Advances in

Operations Research

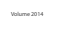

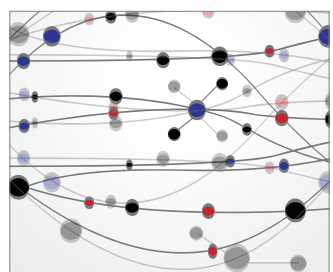

\section{The Scientific} World Journal
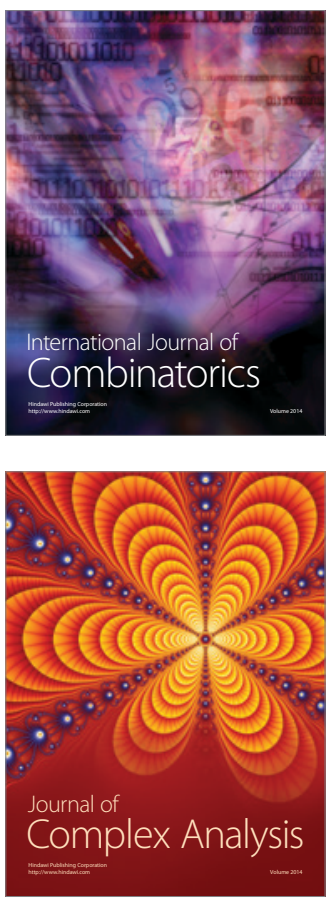

International Journal of

Mathematics and

Mathematical

Sciences
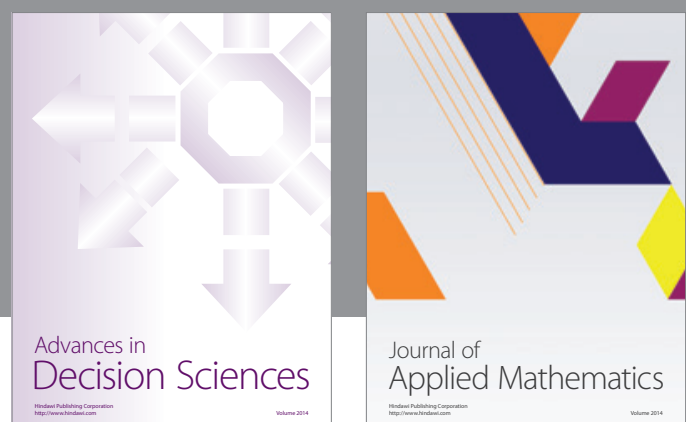

Journal of

Applied Mathematics
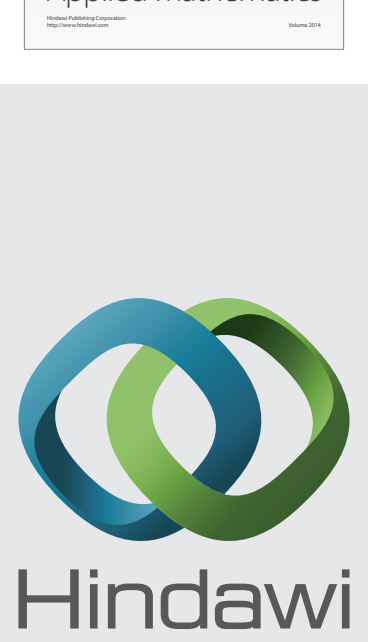

Submit your manuscripts at http://www.hindawi.com
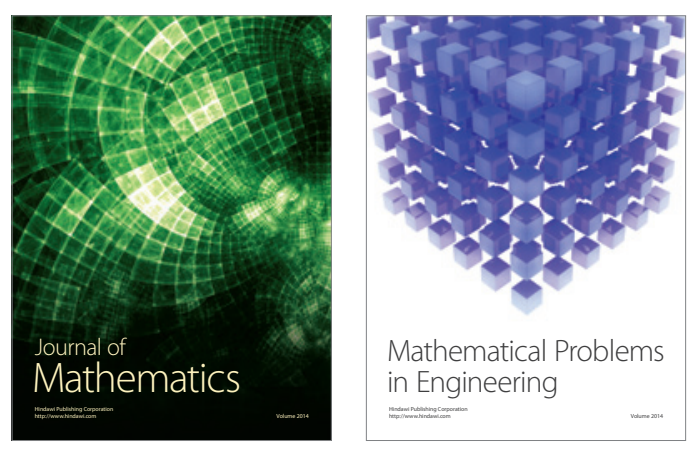

Mathematical Problems in Engineering
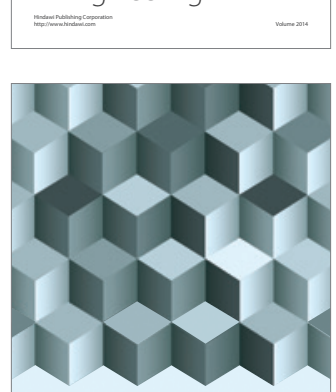

Journal of

Function Spaces
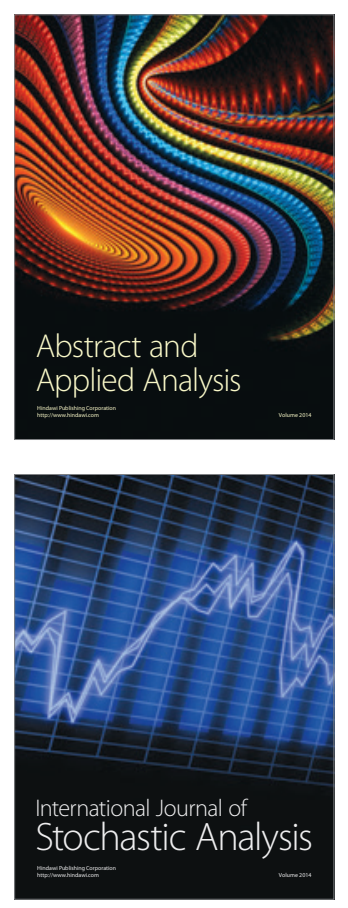

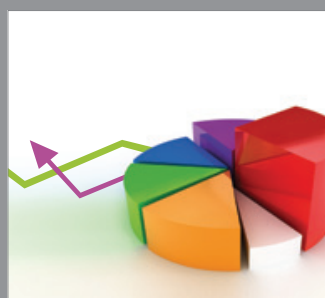

ournal of

Probability and Statistics

Promensencen
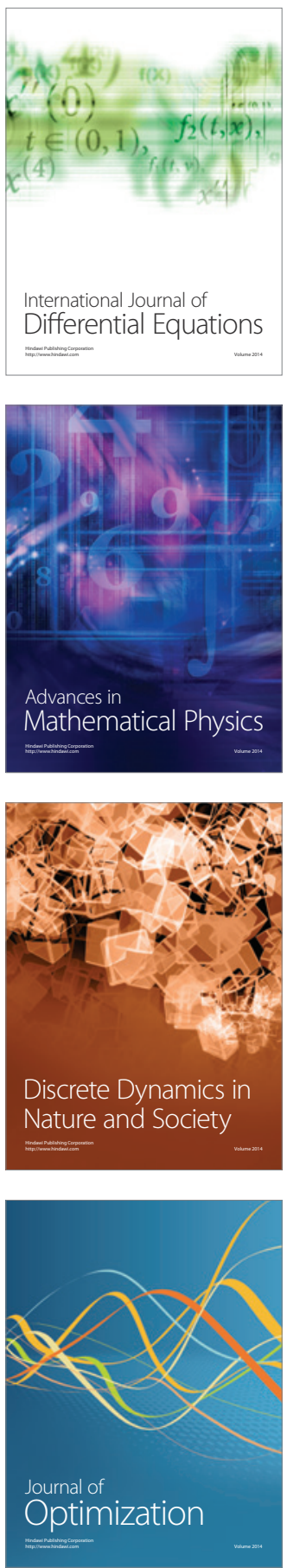